\title{
A PREDICTION APPROACH TO INTRODUCE DEAD-TIME PROCESS CONTROL IN A BASIC CONTROL COURSE
}

\author{
Julio E. Normey-Rico ${ }^{*, 1}$ Teodoro Alamo ${ }^{* * 1}$ \\ Eduardo F. Camacho ${ }^{* *, 1}$ \\ * Depto. de Automação e Sistemas, Universidade Federal \\ de Santa Catarina, Cx.P. 476, 88040-900, Florianópolis, \\ SC,BRASIL.e-mail: julio@das.ufsc.br \\ ** Depto. de Ingeniería de Sistemas y Automática, \\ Universidad de Sevilla, Camino de los Descubrimientos \\ $s / n$, 41092, Sevilla, ESPAÑA. e-mails: \\ \{alamo,eduardo\}@cartuja.us.es
}

\begin{abstract}
This paper presents a methodology to introduce the control of deadtime processes using a simple and intuitive predictive approach. A trivial solution for the control of a process with a dead-time is first proposed. From this strategy the idea of the predictor based controller is derived. Open-loop predictors and closed-loop ones are then used to analyze the obtained solution. A simple tuning of the proposed structure for a first order plus dead-time process together with a polynomial approximation of the dead-time allows to derive a PID controller. Thus, the approach based on the idea of prediction can be used to interpret the use of a PID to control a dead-time process. It is illustrated how the performance of the PID controller is limited by the modelling error introduced in the approximation. The presented approach gives a measurement of the achievable performance. Several simulation examples illustrate the results. Copyright (C)IFAC
\end{abstract}

Keywords: Dead-time compensators, control education, predictors, PID controllers.

\section{INTRODUCTION}

Many processes in industry as well as in other areas exhibit dead-times in their dynamic behavior: central air heaters in buildings; manufacturing supply chains (Schwartz and Rivera, 2006), communication systems (Nemiroff and Bonnell, 2005). In these systems every action that is executed in the manipulated variable of the process will only affect the controlled variable after the process dead-time. Because of this, the analysis and

\footnotetext{
1 The authors acknowledge CAPES-Brazil and MCYTSpain (DPI 2005-04568) for funding this work.
}

design of controllers for dead-time systems is more difficult (Palmor, 1996).

As dead time processes are very common in real word the study of dead-time systems and the design of controllers for these processes is very important and must be included in a basic control course. Two of the most remarkable strategies that use simple concepts to control deadtime process are the Proportional + Integral + Derivative (PID) controllers and the Dead-Time Compensators (DTC). Also in industry these are the most used control algorithms in process control (Takatsu et al., 1998). 
The most popular tuning rules for processes with small dead-times were proposed by Ziegler and Nichols (Ziegler and Nichols, 1942). When the process exhibits a non negligible dead-time, the tuning of the PID is difficult and the performance of the closed-loop limited. Because of this, many effort has been employed in the study and derivation of better tuning rules for PID controllers when controlling processes with time delays (Aström and Hagglund, 1995).

The Smith predictor was the first DTC presented in literature at the end of the 50's (Smith, 1957) to improve the performance of PID controllers for plants with time delay. During the past 20 years numerous extensions and modifications of the SP have been proposed: to improve the regulatory capabilities of the sP for measurable or unmeasurable disturbances; to allow its use with integrative plants or to facilitate the tuning in industrial applications (Palmor, 1996).

Traditionally, in a basic control course, the control of dead-time systems is first analyzed using PID controllers and after that, dead-time compensation is introduced. This paper presents a different approach where the dead-time compensation is considered as the natural solution for the control of a dead-time process. This prediction approach allows to derive the PID control of these processes and the achievable performance of the controllers.

From a pedagogical point of view the proposed approach gives to the students a better comprehension of the problems associated to the control of dead-time systems. The presented approach cannot be applied for unstable or high order systems with delay. These points are discussed in details in advanced control courses.

The article is organized as follows. Next section introduce some typical examples that illustrate the effect of the dead-time and can be used as introductory problems for the study of the subject. Section 3 presents the control problem while section 4 introduces the concept of "ideal solution" and the prediction approach to solve the proposed problem. Section 5 derives a PID tuning rule for dead-time process base on the previous ideas. The paper ends with the conclusions.

\section{INTRODUCING DEAD-TIME SYSTEMS}

Dead time is a natural phenomenon in several processes and could be easily introduced in process modelling using simple concepts. A simple classification of the principal dead-time causes is:

- mass and/or energy transportation

- communications lags

- processing time in analyzers
- accumulation of low order systems.

Examples of the first group are for instance a central air heater of a building or the simple water heater installed in a house. Cheap laboratory pilot plants with these characteristics can be easily created to be used in illustrative experiments.

Communication dead-time is inevitable in the remote control of a vehicle, as for instance a moon mobile robot. When instructions have to be transmitted from the earth platform to the mobile robot a dead-time composed of the time spent by the signal to travel from the earth to the moon and the other way round must be considered in the communication system. Here, the dead time depends on the velocity of the signal and the distance between the earth and the moon (Nemiroff and Bonnell, 2005).

Many processes in the chemical industry are good examples of the third group, where analyzers, that need some time to process the analysis, are used to obtain some properties of the products. This is the case for instance in a continuous stirred tank reactor where the outlet concentration of product has to be controlled and an analyzer is used to measure the desired process variable (Ogunnaike and Ray, 1994).

Using simulation tools or simple processes in the lab it is possible to create some environments to introduce, in a virtual or real experiment, the effects produced by the dead-time in the process behavior. Although the dead-time in the previous classification has different causes and origins, the first three are "real" dead-times that can be easily understood. The last one is an apparent dead-time caused by the accumulative effect of lags of several dynamic systems and needs a more elaborated description.

A set of interconnected tanks is a simple case to be used in the real or virtual experiment. Consider, for instance, a set of $n$ equal cylinder atmospheric tanks where the output flow of tank $i\left(F_{O}^{i}\right)$ is used to feed tank $i+1$, that is, the input flow of tank $i+1$ is $F_{I}^{i+1}=F_{O}^{i}$. When the tank levels are close to an operating point the dynamic behavior of the level in each tank $H^{i}$ can be modelled by a linear system:

$$
\begin{aligned}
A \frac{d H^{i}}{d t} & =F_{I}^{i}-F_{O}^{i} \\
F_{O}^{i} & =K H^{i},
\end{aligned}
$$

where $A$ is the area of the base of the tank and $K$ is a constant that depends on the tank characteristics. Thus, the transfer function relating the input flow in tank $i$ and its level is:

$$
H^{i}(s)=\frac{1 / K}{T s+1} F_{I}^{i}, \quad T=A / K .
$$


Thus, the transfer function relating $F_{I}^{1}$ with the level in tank $n\left(H^{n}\right)$ is:

$$
H^{n}(s)=\frac{K_{e}}{(T s+1)^{n}} F_{I}^{1}, \quad K_{e}=1 / K .
$$

As a numerical example consider the step response of a system with $n=4, K_{e}=2$ and $T=1$ seconds shown in Figure 1. As can be seen an apparent dead-time of approximately 2 seconds appears in the step response of the system.

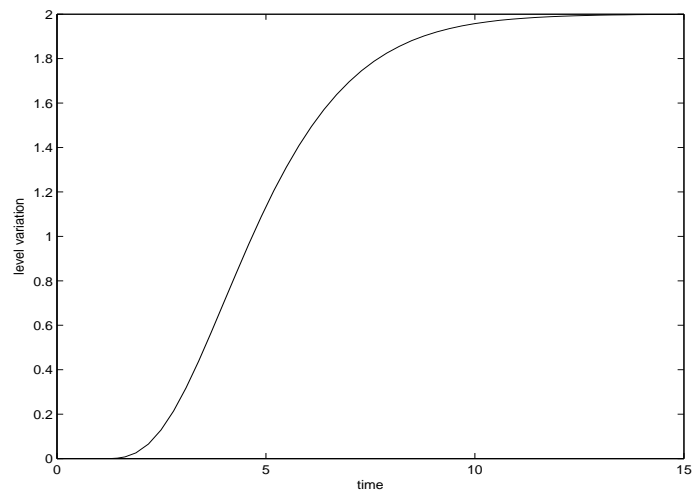

Fig. 1. Step response of the fourth tank level.

An interesting low cost process to be used in the lab for different experiments with dead-time systems is shown in Figure 2. The water is heated in the tank using an electric resistance and driven by a bomb through a long tube to the output of the system. The control input is the power

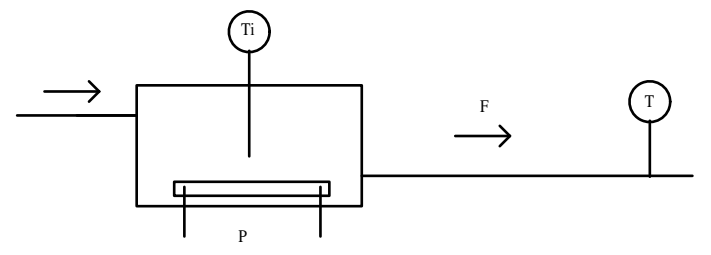

Fig. 2. The heated tank and the long tube.

$P$ in the resistance and the plant output is the temperature $T$ at the end of the tube. A linear model of the process could be obtained using a simple step-test identification procedure near an operation point $P_{0}, T_{0}$. Figure 3 shows $T$ when a step is applied in $P$. In this simulated situation the power $P$ (in dashed line) changes from $40 \%$ to $50 \%$ at $t=1$ and the temperature increases from $55 \%$ to $65 \%$ (in solid line). Note that the temperature inside the tank $T_{i}$ (in dotted-dashed line) starts growing at $t=1 \mathrm{~s}$ while the temperature at the end of the tube only reacts at $t=6 \mathrm{~s}$, then there is a dead-time of 5 seconds due to the mass transportation. Thus, it is possible to relate the two temperatures considering the signal relation $T_{i}(t)=T(t+5), t \geq 1$. Using the Laplace transform $\mathcal{L}\{x(t+L)\}=e^{L s} \mathcal{L}\{x(t)\}$, it is possible to represent the dynamic relation between the variations on $T_{i}, T$ and $P\left(\Delta T_{i}, \Delta T\right.$ and $\left.\Delta P\right)$ as:

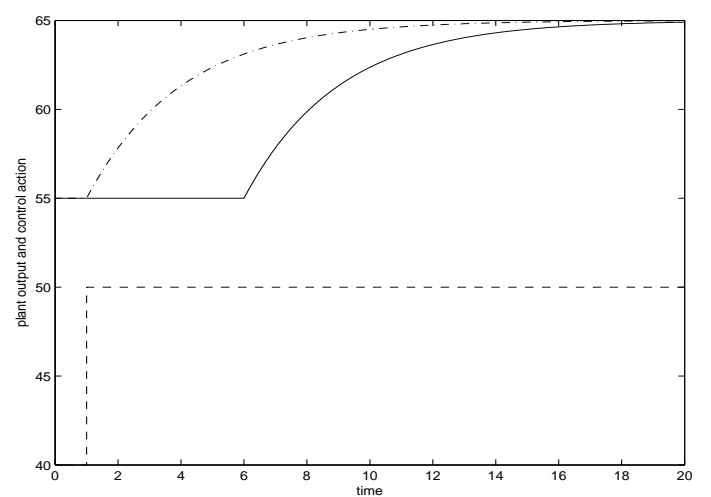

Fig. 3. Step response of the system: $T_{i}$ (dotteddashed), $T$ (solid) and $P$ (dashed).

$$
G(s)=\frac{\Delta T_{i}(s)}{\Delta P(s)} \Rightarrow \frac{\Delta T(s)}{\Delta P(s)}=G(s) e^{-L s} \quad L>0 .
$$

In this simple process, when a constant flow of the water $F$ is used, the dead time $L$ can be estimated using $F$ and the volume of the tube $V$ as $L=\frac{V}{F}$. Thus, for the experiments different dead-times and time transients can be used if the flow could be manipulated.

Both in the simulations and the experiments, several temperature sensors are used, one for $T_{i}$ and the others in different positions of the tube. With this configuration it is possible to change the dead-time in the measurement of $T$ without changing the dominant time constant of $G(s)$.

\section{THE CONTROL PROBLEM}

After understanding the effect of the dead-time in the open-loop operation the difficulties in the control of a dead-time process are introduced. For this purpose, the heated tank with the long tube is used.

The idea here is simple. A previous experiment was proposed in which a proportional + integral (PI) controller was used to control $T_{i}$, obtaining the response shown in Figure 4 (solid), when a change of $1 \%$ is introduced in the set-point at $t=1 \mathrm{~s}$. Using the same tuning the controller is then used to control the temperature $T$ at the end of the tube. The obtained behavior is very oscillatory, as can be seen in Figure 4 (dashed).

The students are asked to re-tune the PI controller to obtain a non-oscillatory behavior, as the one in Figure 4 (dashed-dotted). Note that the oscillations disappear and the the price for this is a slower step response.

This example allows to understand that the the dead-time has then two effects. The first one is the physical constrain that does not allow the temperature to react until $L$ seconds after the change in $P$. Nothing can be done about this. The 

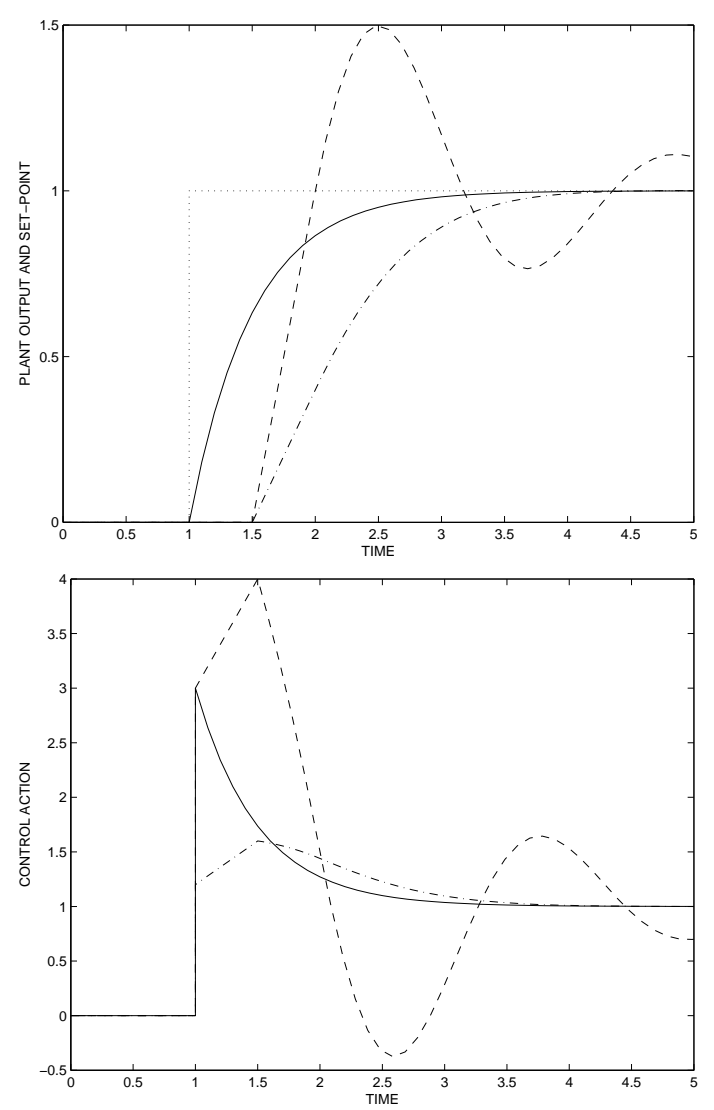

Fig. 4. Step response and control action for $K_{c}=3$ and $T_{i}=1.5$ with $L=0$ (solid); $K_{c}=3$ and $T_{i}=1.5$ with $L=0.5$ (dashed) and $K_{c}=1.2$ and $T_{i}=1.5$ with $L=0.5$ (dashed-dotted).

second effect is the deterioration of the closed-loop transient after the dead-time. However, as it will be shown, there are some simple control structures that allow to improve the closed-loop performance of these systems.

\section{THE IDEAL SOLUTION}

From this point the students have understood that if the temperature sensor is installed at the beginning of the tube a simple PI controller could be tuned to achieve a good performance in $T_{i}$. They are then induced to observe the two temperature signals simultaneously during the closed-loop operation. As the tube is thermally isolated $T$ has the same behavior as $T_{i}$ after the dead-time. They then understand that this is obviously the ideal solution for a dead-time process: "to feed the plant output without the dead-time". Figure 5 shows this ideal situation in a general case, where the controller $C(s)$ is tuned using only $G(s)$ and the real output $y(t)$ is the output of $G(s)\left(y_{1}\right)$ delayed $L$ unities of time $\left(y(t)=y_{1}(t-L)\right)$. In this situation the dead-time has no effect in the closed-loop transients, as the closed loop transfer function is:

$$
\frac{Y}{R}=\frac{C(s) G(s) e^{-L s}}{1+C(s) G(s)} .
$$

The real implementation of this solution is, in

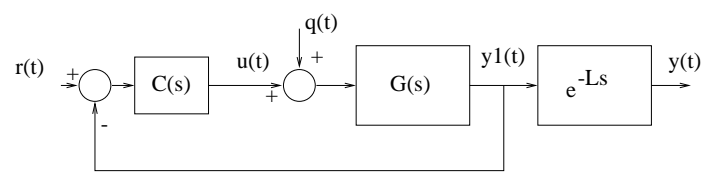

Fig. 5. "Ideal" control for dead-time processes.

general, not possible in practice principally because the sensor cannot be installed in the desired position and/or the process has an apparent and not a real dead-time caused by mass transportation, as is the case of the series of tanks presented in section 2 .

A simple solution for this problem is then suggested using the idea of prediction and is applied to the temperature process. Considering that $G_{n}(s)$ is a free dead-time model of the plant, that has a behavior represented by $P(s)=G(s) e^{-L s}$, it is possible to feed the output of this model to the controller as shown in Figure 6. In this structure

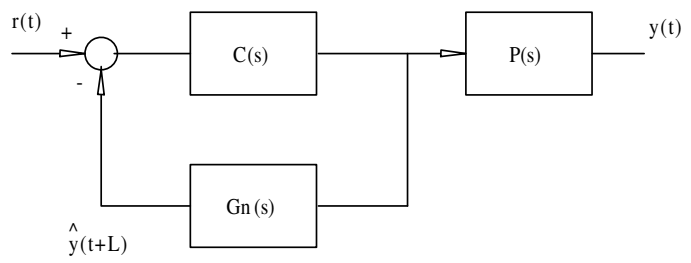

Fig. 6. Open loop predictor.

if $G_{n}(s)=G(s)$ the primary controller $C(s)$ can be tuned considering only $G(s)$ and the obtained closed loop performance will be the same as in the ideal case: $\frac{Y}{R}=\frac{C(s) G(s) e^{-L s}}{1+C(s) G(s)}$.

The equivalent controller for this system is:

$$
C_{e q}(s)=\frac{C(s)}{1+C(s) G_{n}(s)},
$$

that operates in open-loop. This strategy is known as open loop predictor based controller and it is clear that cannot be used in practice because the controller does not "see" the effect of the disturbances and also the model mismatch is not taken into account. At this point students are asked to test the open predictor in the plant and verify this behavior.

From this study, the idea of the closed-loop predictor is introduced naturally as a better solution for this problem. Thus, the Smith predictor (SP) structure (Smith, 1957) is proposed and analyzed for a stable process. This structure is shown in Figure 7. In this strategy, the prediction at time $t$ is computed by the use of a model of the plant without dead-time $\left(G_{n}(s)\right)$ and, in order to correct the modelling errors, the difference between the 


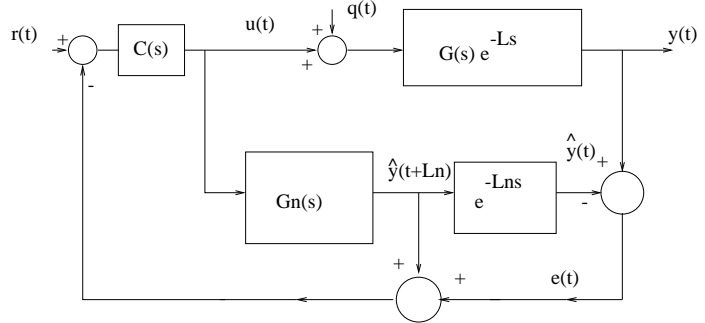

Fig. 7. Smith Predictor structure.

output of the process and the model (including the dead-time $\left.P_{n}(s)=G_{n}(s) e^{-L_{n} s}\right)$ is feed back, as can be seen in the scheme. With this structure, if there are no modelling errors or disturbances, the error between the current process output and the model output will be null and the controller could be tuned, at least in the nominal case, as if the plant had no dead-time. Thus, in the nominal case this structure gives the same performance as the ideal solution but can also operate in the real case when correctly tuned. The fundamental properties of the SP are studied latter but at this moment the students are induced to use this strategy in the heated tank, first in simulation and then in the real process.

In the simulations two different dead-times are used: $L=0.5$ and $L=5$. Considering perfect modelling and the same PI they observe how the predictor can cope with the dead-time. Figure 8 shows the closed loop response for the two cases, that have, after the dead-time, the same transient. They also note that the control action is the same in the two cases because the controller acts as if the process had no dead-time. When applying this strategy to the real plant, that has a dynamic behavior different to the model, the obtained responses are not ideal but the students can observe the effect of the closed-loop prediction structure. Also they can analyze the effect of the tuning in the closed-loop stability. In following lessons, that are not discussed here, the students undertake the complete study of the robustness and disturbance rejection properties of the SP.

This analysis shows that the control of deadtime processes could be solved efficiently using predictive control structures. At this point, the objective of the work is to introduce a tuning of a PID controller to improve the closed-loop behavior obtained with the PI.

\section{PID TUNING: THE PREDICTION APPROACH}

The idea of prediction can be used to interpret the use of a PID to control a dead-time process. In previous lessons PID controllers were introduced to control dead-time free processes. The PID is con-

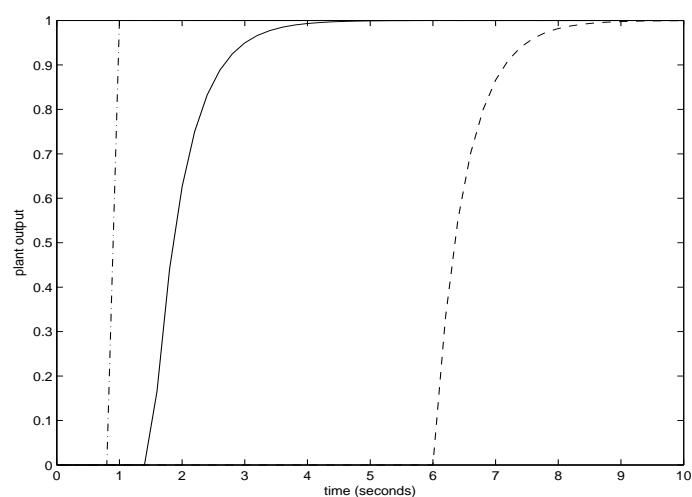

(a)

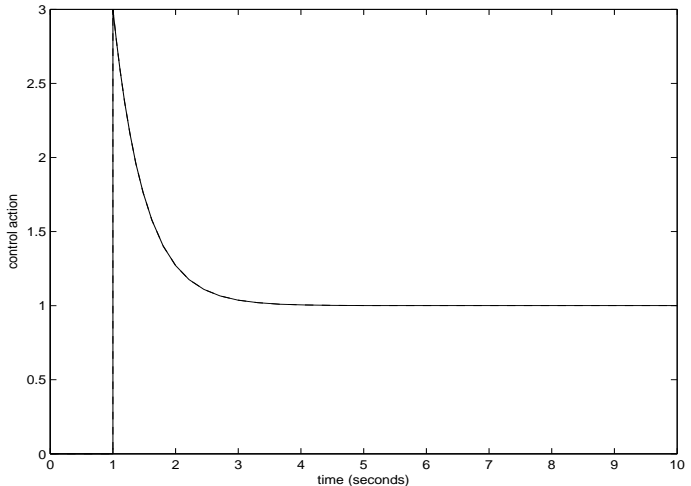

(b)

Fig. 8. Step response and control action for the SP with $K_{c}=3$ and $T_{i}=1.5$ for a step change in the set-point (dashed-dotted line): (a) $L=0.5$ (solid line); (b) $L=5$ (dashed line).

sidered as a second order controller with transfer function:

$$
C_{p i d}(s)=K_{C} \frac{1+s T_{1}+s^{2} T_{2}}{s\left(1+s T_{f}\right)},
$$

tuning parameters $K_{C}, T_{1}, T_{2}$ and $T_{f}$ defining the low pass filter of the derivative action.

A tuning rule for the PID can be derived in the case of dead-time processes using the idea of prediction. First a SP is tuned and then the obtained controller is approximated by a PID.

From the block diagram of the SP an equivalent controller can be computed as shown in Figure 9.

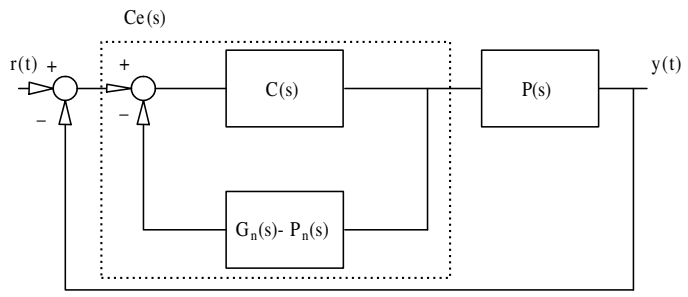

Fig. 9. Equivalent diagram of the SP.

In this structure $C_{e}(s)$ can be computed as: 


$$
C_{e}(s)=\frac{C(s)}{1+C(s)\left(G_{n}(s)-P_{n}(s)\right)},
$$

where a simple model is assumed $P_{n}(s)=\frac{K_{p} e^{-L s}}{1+T s}$, $G_{n}(s)=\frac{K_{p}}{1+T s}$ and $C(s)=\frac{K_{c}\left(1+T_{i} s\right)}{T_{i} s}$. The PI can be tuned using the dead-time free model. A simple solution is to choose $T_{i}=T$ and use $K_{c}$ to adjust the speed of the set point response given by the time constant $T_{o}=\frac{T}{K_{c} K_{p}}$ (Hagglung, 1996; Ingimundarson and Hagglund, 2001). In this case:

$$
C_{e}(s)=\frac{\frac{K_{c}(1+T s)}{T s}}{1+\frac{K_{c} K_{p}}{T s}\left(1-e^{-L s}\right)} .
$$

Using a first order rational approximation of the dead-time $e^{-L s} \cong \frac{1-s L / 2}{1+s L / 2}$ this controller can be approximated by a PID as the one in equation 1 :

$$
C_{p i d}(s)=\frac{K_{c}(1+T s)(1+0.5 L s)}{T s\left(1+0.5 L s+\frac{K_{c} K_{p} L}{T}\right)}
$$

where the tuning of $K_{c}$ will define the closed loop performance, that is limited by the modelling error introduced in the approximation.

The following step in the solution of the problem is to tune the PID controller for different values of $L$ and evaluate the closed-loop performance. The results obtained in the simulations and in the experiments are then analyzed using a frequency domain approach that shows the effect of the approximation error in the performance index of the controller.

The open loop transfer function of the system for real case $H(s)=C_{e}(s) P(s)$ and for the approximated case $H_{a}(s)=C_{\text {pid }}(s) P(s)$ are computed using $L$ and $T_{o}$ :

$$
\begin{gathered}
H(s)=\frac{e^{-L s}}{T_{o} s+1-e^{-L s}} \\
H_{a}(s)=\frac{e^{-L s}(1+0.5 L s)}{T_{o} s\left(1+L / T_{o}+0.5 L s\right)} .
\end{gathered}
$$

Thus, the relation between $L$ and $T_{o}$ determines the error of the approximation. The students are asked to compute the phase margin (PM) and gain margin (GM) using $H$ and $H_{a}$. Thus, they verify that as the relation $L / T_{o}$ increases, the differences between the ideal and approximate systems increases and the approximation is not valid. As the deterioration in the performance is directly associated to the PM, for a given deadtime it is possible to define, for instance, the achievable $T_{o}$ for a non-oscillatory closed-loop response.

In next lessons these ideas and concepts are related to the robust analysis and tuning of the PID and DTC.

\section{CONCLUSIONS}

This paper presents a methodology to introduce the control of dead-time processes using a simple and intuitive predictive approach. A simple process that can be used in a virtual or real laboratory is introduced to present the analysis and control of dead-time systems. The process allows the use of the natural idea of anticipative measurement that allows a simple introduction of the predictor in the control context. A rational approximation of the dead-time allows to derive a PID controller from this predictor based controller. The performance of the PID controller is limited by the modelling error introduced in the approximation, which allows to present a measurement of the achievable performance of the PID. Several simulation examples illustrate the results and some comments related to the use of this methodology in a basic control course are also presented.

\section{REFERENCES}

Aström, K.J. and T. Hagglund (1995). PID Controllers: Theory, Design and Tuning. Instrument Society of America.

Hagglung, T. (1996). An industrial dead-time compensating PI controller. Control Engineering Practice 4(6), 749-756.

Ingimundarson, A. and T. Hagglund (2001). Robust tuning procedures of dead-time compensating controllers. Control Engineering Practice 9, 1195-1208.

Nemiroff, Robert and Jerry Bonnell (2005). Nasa web site. http://www.astronet.ru.

Ogunnaike, B.A. and W.H. Ray (1994). Process Dynamics, Modeling, and Control. Oxford University Press.

Palmor, Z.J. (1996). The Control Handbook. Time Delay Compensation: Smith Predictor and its Modifications. CRC Press and IEEE Press.

Schwartz, Jay D. and D. E. Rivera (2006). Control relevant demand modeling for supply chain management. In: Proceedings of 14th IFAC Symposium on System Identification (SYSID 2006). Newcastle, Australia.

Smith, O. J. M. (1957). Closed control of loops with dead-time. Chem. Eng. Progress 53, 217-219.

Takatsu, H., T. Itoh and M. Araki (1998). Future needs for the control theory in industries. report and topics of the control technology survey in japanese indsutry. Journal of Process Control 8, 369-374.

Ziegler, J.G. and N.B. Nichols (1942). Optimum Settings for Automatic Controllers. Trans. ASME 64, 759-768. 